\title{
Efficiency of Gymnema sylvestre-derived gymnemic acid on the restoration and improvement of brain vascular characteristics in diabetic rats
}

\author{
NICHAWADEE SANDECH, RAWIPA JANGCHART, MANARAS KOMOLKRIENGKRAI, \\ PIYAKORN BOONYOUNG and WIPAPAN KHIMMAKTONG
}

Department of Anatomy, Faculty of Science, Prince of Songkla University, Hat Yai, Songkhla 90110, Thailand

Received October 7, 2019; Accepted September 7, 2021

DOI: $10.3892 /$ etm.2021.10855

\begin{abstract}
The brain is a vital organ that requires a constant blood supply. Stroke occurs when the blood supply to specific parts of the brain is reduced; diabetes is an autonomous risk factor for stroke. The present study aimed to investigate the potential vascular protective effect of gymnemic acid (GM) by assessing the morphological changes of microvasculature, along with VEGFA and angiopoietin-1 (Ang-1) protein expression in the brains of diabetic rats. Rats were divided into five groups, including control, gymnemic control rats (CGM), rats that were rendered diabetic by single injection of $60 \mathrm{mg} / \mathrm{kg}$ streptozotocin (STZ), diabetic rats treated with $400 \mathrm{mg} / \mathrm{kg}$ $\mathrm{GM}(\mathrm{STZ}+\mathrm{GM})$ and diabetic rats treated with $4 \mathrm{mg} / \mathrm{kg}$ glibenclamide (GL; STZ + GL). After 8 weeks, brain tissues were collected to examine the three-dimensional morphology of the anterior cerebral arteries by vascular corrosion casting. Western blotting was performed to determine VEGFA and Ang-1 expression. Cerebral arteries, arterioles and capillaries were depicted the diameter, thickness and collagen accumulation of the wall, and the results demonstrated narrow diameters, thickened walls and collagen accumulation in the STZ group. After receiving GM, the histopathological changes were similar to that of the control group. Through vascular corrosion casting and microscopy, signs of vessel restoration and improvement were exhibited by increased diameters, and healthy and nourished arterioles and capillaries following treatment with GM. Furthermore, VEGF expression and Ang-1 secretion decreased in the STZ + GM group compared with STZ rats. The results of the present study revealed that GM treatment decreased blood vessel damage in the brain, suggesting that it may be used as a therapeutic target for the treatment of diabetes.
\end{abstract}

Correspondence to: Dr Wipapan Khimmaktong, Department of Anatomy, Faculty of Science, Prince of Songkla University, Preclinic Building, 15 Kanjanavanich Road, Hat Yai, Songkhla 90110, Thailand E-mail: wipapan.k@psu.ac.th

Key words: diabetes mellitus, streptozotocin, gymnemic acid, brain, vascular corrosion cast

\section{Introduction}

Diabetes mellitus (DM) is a metabolic dysfunction disease that is caused by insufficient insulin action or ineffective insulin production. Insulin is a naturally occurring hormone produced by the $\beta$ cells of pancreatic islets, which helps the body utilize glucose for energy (1). Abnormal insulin levels lead to hyperglycemia, which causes acute or chronic complications in patients with diabetes, such as vascular disease (2). The brain is an organ of the central nervous system (CNS) that is most responsive to changes in oxygen and metabolites in humans and animals. It maintains neuronal function and control over other organs of the body. Because the nervous system and blood circulation are associated, disturbances of cerebral blood flow (CBF) lead to changes in neural function (2). DM has been associated with pathological changes in the CNS, which can lead to cognitive and affective deficits as well as increased risk of vascular complications in the brain (3).

The circle of Willis connects the anterior and posterior cerebral circulations. The anterior circulation of the brain derives from the bilateral internal carotid arteries and branches of the common carotid arteries; the posterior circulation derives from the bilateral vertebral arteries and branches of the subclavian arteries, which combine to form the posterior cerebral artery (4). The anterior cerebral circulation supplies blood to the anterior portion of the brain. It is supplied by the internal carotid artery (ICA) and its branches: The anterior cerebral artery (ACA) and the middle cerebral artery (MCA) (4). The ICA supplies oxygenated blood to most midline portions of the frontal lobe and superior medial parietal lobe. It is the most important blood supply of the cerebral circulation from the circle of Willis (5). The MCA receives $80 \%$ of the carotid blood flow, whereas the ACA receives $20 \%$. Each branch of the anterior circulation supplies the cortex and the basal surface of the brain, providing blood to deep structures, such as the anterior hypothalamus, basal ganglia and internal capsule (6).

Diabetes mellitus increases the risk of cardiovascular and cerebrovascular diseases (7). Furthermore, it has been demonstrated to diminish angiogenesis in peripheral vascular beds (7). VEGFA and Ang-1 are highly effective inducers of endothelial proliferation; they also promote hyperplasia, tortuosity and a decreased lumen diameter. High blood 
glucose conditions result in brain hypoxia-induced angiogenesis through the upregulation of hypoxia-inducible genes, such as hypoxia-inducible factor-1 (HIF-1). The vascular inner wall affects blood pressure and, thus, the perfusion of nutrients and blood cells that control the delivery of oxygen and immunological surveillance. Several factors, including inflammatory cytokines (TNF- $\alpha$ and IL-1 $\beta$ ) influence endothelial cell luminal integrity as a result in capillaries tube regression, a critical pathological process regulating human disease. Investigating the mechanisms and the factors that control, maintain and regulate lumen size and diameter will increase our understanding of pathophysiological processes. Lumen diameter is tightly regulated by several factors (8). For example, VEGFA and its receptors increase lumen formation and decrease lumen diameter through the proliferation of myointimal cells and collagen deposition (8,9). Ang-1 stabilizes the nascent vessel (10).

An increased expression of VEGFA is caused by local ischemic conditions and the activation of hypoxia-induced factor (HIF)-1 $\alpha$ (7). Brain angiogenesis complicates several brain functions following the release of a number of growth factors from the endothelial cells of the brain, including VEGFA (11). VEGFA is a protein with increase vascular permeability activity during angiogenesis, which regulates the formation and maintenance of blood vessel structures and is considered crucial for the progression of several pathology and diseases, such as cancer (11). VEGFA serves an important role in mediating diabetic vasculopathy and microvascular permeability (12). As a potent vasodilator, VEGFA is regarded as a survival factor for endothelial cells (12). Angiopoietins also serve important roles in vascular development and angiogenesis. Ang-1 is a member of the angiopoietin family. Ang-1 and its receptor, Tie-2, are important for the regulation of angiogenesis. Tie- 2 receptors are predominantly expressed on endothelial cells (13). Furthermore, the Tie2/Ang-1 signaling pathway serves a key role in the latter stages of improvement of vascular dysfunction, remodeling and stabilizing vessels, suppressing plasma leakage, inhibiting vascular inflammation and preventing endothelial cell death (13). Ang-1 serves a crucial role in mediating reciprocal interactions between endothelium, surrounding matrix and mesenchyme; it is also involved in vascular maturation and stability (10). Endothelial budding is facilitated by vasodilation and the loosening of endothelial cell contacts (14). The pre-existing vessels will exhibit leakiness, which allows extravascular plasma protein and extracellular matrix components to migrate out from endothelial cells through the extracellular matrix toward an angiogenic stimulus, such as VEGFA, that can promote growing and survival of new blood vessels (14).

To the best of our knowledge, the effects of gymnemic acid (GM) on VEGFA or Ang-1 have not yet been reported in a rat model of diabetes-induced stroke.

Gymnema sylvestre (Asclepiadaceae; GS) is a potent antidiabetic plant that is used in folk, ayurvedic and other alternative medicines (15) to treat of asthma, eye complaints such as cornea opacity, inflammations, family planning by treating amenorrhea and snakebites (15). GS has been reported to exhibit antimicrobial, antihypercholesterolemic and hepatoprotective activities (15). GM is a mixture of oleanane-type triterpene saponins extracted from the leaves of GS (16). GM is known to suppress sweetness, whereby after chewing the leaves, sweetened solutions with sucrose taste like water (17). A previous study demonstrated that GM decreases blood glucose levels and increases plasma insulin levels in diabetic rats (17). The molecular structure of GM is similar to that of glucose; it can bind to the glucose receptors on the taste buds of the tongue and prevent its activation by sugar molecules from food and reducing its sugary taste (18). Additionally, the absorptive external layers of the intestine exhibit receptors for GM molecules that prevent the absorption of sugar molecules, lowering blood glucose levels (19). GM improves glucose and lipid metabolism in type two DM model rats, enhances glucose uptake by regulating the amelioration of endoplasmic reticulum stress and insulin transduction in insulin resistant HepG2 cells (20), and inhibits GAPDH during glycerol metabolism in rabbits (21).

The present study aimed to investigate the restoration and improvement of GM on the blood vessels of the anterior cerebral circulation that supply the majority of blood to the brain, including arteries (ICA, MCA and ACA) and small vessels (arterioles and capillary network) of the anterior cerebral circulation, which provide crucial blood supply into the cerebral circulation via the circle of Willis. The hypothalamus is an area of the brain that is surrounded by branches of arteries that originate near the stump of the circle of Willis (4). Therefore, this area of the brain was selected for assessment in the present study. In addition, the protein expression levels of VEGFA and Ang-1 in the hypothalamus were analyzed to determine their association with affecting angiogenesis or formation of new blood vessels in diabetic rat's brain.

\section{Materials and methods}

Animals. All experimental protocols were reviewed and approved by the Animal Ethics Committee of the Prince of Songkla University (Songkhla, Thailand). A total of 80 male Wistar rats (weight, 200-250 g; age, 8 weeks) were purchased from Nomura Siam International Co., Ltd. Animals were housed under standard laboratory conditions under a 12-h light/dark cycle, with good lighting, moderate temperature $\left(25+2^{\circ} \mathrm{C}\right)$ with lights on at 7:00 a.m. and humidity $(50 \pm 10 \%)$ and adequate ventilation in a hygienic environment. They were fed ad libitum with standard rat chow containing protein, carbohydrate, fat, vitamins and minerals.

Induction and assessment of diabetes. Experimental diabetic rats were intraperitoneally injected with a single $60 \mathrm{mg} / \mathrm{kg}$ dose of streptozotocin (STZ; Sigma-Aldrich; Merck KGaA) dissolved in $0.1 \mathrm{~mol} / 1$ citrate buffer (Sigma-Aldrich; Merck KGaA). Control rats were injected with $0.1 \mathrm{~mol} / \mathrm{l}$ citrate buffer alone. Blood glucose levels were measured 3 days after STZ injection and persistent hyperglycemia was confirmed 1 week after STZ injection, using an Accu-Chek Active ${ }^{\circledR}$ one-touch glucometer and test strips (Roche Diagnostics $\mathrm{GmbH}$ ). Rats with blood glucose levels $>250 \mathrm{mg} / \mathrm{dl}$ were considered diabetic. The rats were randomly divided into five groups (Fig. 1). (Normal control rats that received a balanced standard diet (control group; $\mathrm{n}=10$ ); 1B) gymnemic control rats (CGM group; $n=10$ ) that received a balanced standard diet supplemented with $400 \mathrm{mg} / \mathrm{kg}$ GM (Xi'an Guanyu 


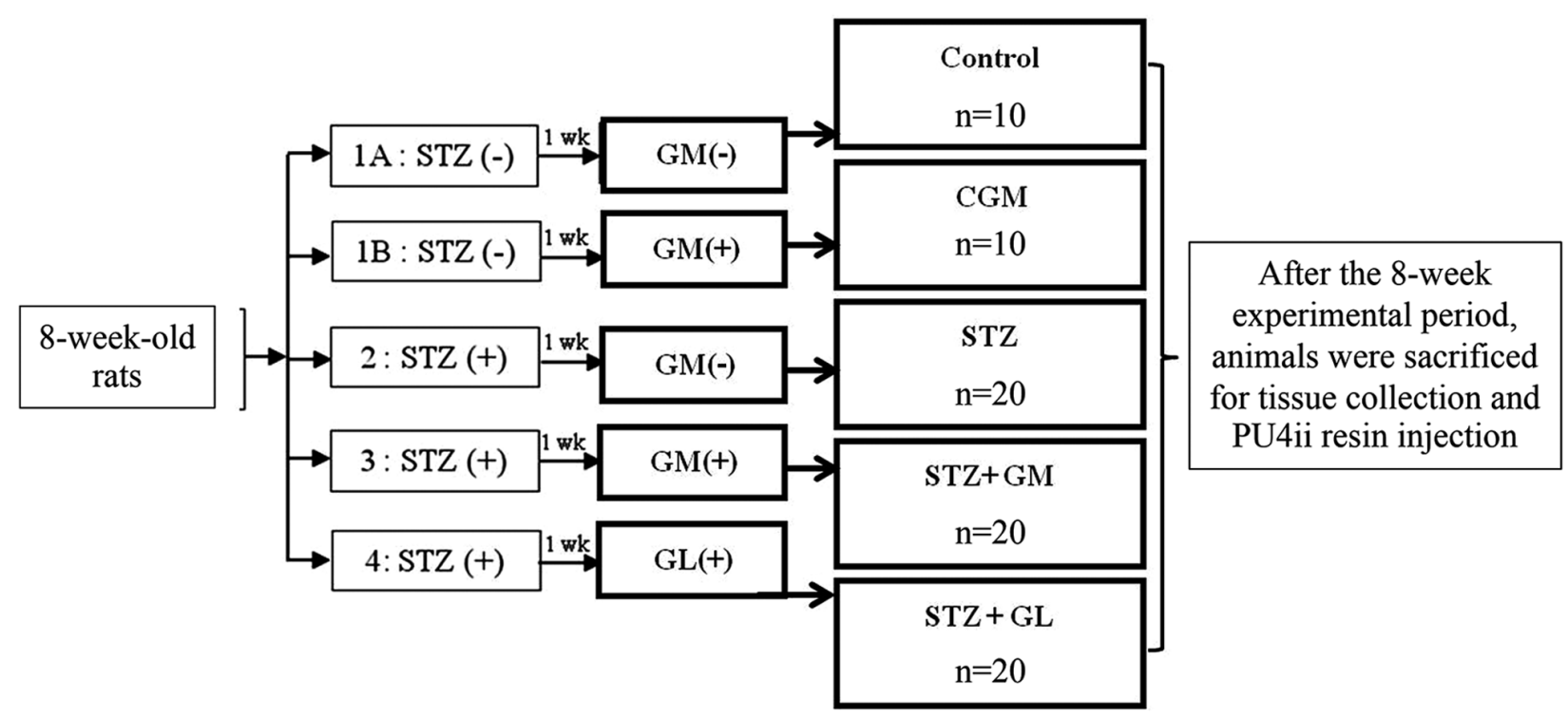

Figure 1. Experimental design. The rats were randomly divided into five groups; $1 \mathrm{~A}$, Normal control rats that received a balanced standard diet; 1B, gymnemic control rats (CGM) that received a balanced standard diet supplemented with GM; 2, diabetic rats that received a balanced standard diet and intraperitoneally injected with $60 \mathrm{mg} / \mathrm{kg} \mathrm{STZ} \mathrm{(STZ);} \mathrm{3,} \mathrm{diabetic} \mathrm{rats} \mathrm{that} \mathrm{received} \mathrm{a} \mathrm{balanced} \mathrm{standard} \mathrm{diet} \mathrm{supplemented} \mathrm{with} \mathrm{GM} \mathrm{(STZ} \mathrm{+} \mathrm{GM)} \mathrm{and} \mathrm{4,} \mathrm{diabetic} \mathrm{rats} \mathrm{that}$ were treated with glibenclamide (STZ + GL). After the 8-week experimental period, animals were sacrificed for tissue collection and PU4ii resin injection. Wk, week; GM, gymnemic acid; CGM, gymnemic control; STZ, streptozotocin; GL, glibenclamide.

Bio-Tech Co.) (22) (purified $>75 \%$ via high-performance liquid chromatography analysis) in $0.5 \mathrm{ml} 0.5 \%$ Tween- 80 solution (Sigma-Aldrich; Merck KGaA); 2) diabetic rats that received a balanced standard diet and intraperitoneally injected with $60 \mathrm{mg} / \mathrm{kg} \mathrm{STZ} \mathrm{(STZ} \mathrm{group;} \mathrm{n}=20$ 3) diabetic rats that received a balanced standard diet supplemented with $400 \mathrm{mg} / \mathrm{kg} \mathrm{GM}$ in $0.5 \mathrm{ml} 0.5 \%$ Tween-80 solution (STZ + GM group; $n=20$ ); and 4) diabetic rats that were treated with $4 \mathrm{mg} / \mathrm{kg}$ glibenclamide (GL) (23) in $0.5 \mathrm{ml} 0.5 \%$ Tween- 80 solution ( $\mathrm{STZ}+\mathrm{GL}$ group; $\mathrm{n}=20$ ). All animals were clinically observed, such as weight loss, dehydration, cataracts, drowsiness, and diabetic coma and weighed on a weekly basis. No deaths were observed following supplementation with GM by being fed only one time per day for a total of 8 weeks. Following animal sacrifice, brain tissue was collected. Half of the rats in each group [n=5 (C and CGM groups) $n=10$ (STZ, GM and GL groups)], tissue were used for H\&E staining and western blot analysis. The other half of rats in each group were injected with resin for vascular corrosion casting combined with scanning electron microscopy. The experimental rats were euthanized by an excessive dose of sodium pentobarbital (200 mg/kg; intraperitoneal injection). Death was confirmed by observation of cardiac and respiratory arrest or fixed and dilated pupils.

Histological preparation for Masson's trichrome staining. To analyze any histological changes and measure the lumen diameter of the ICA, MCA and ACA, brain tissue from all groups was dissected and immediately fixed in $10 \%$ formalin for $6 \mathrm{~h}$ at room temperature. Tissue sections were dehydrated in a descending ethanol series $(70,80,90,95$ and $100 \%)$, with twice in succession, each lasting $1 \mathrm{~h}$. Prior to paraffin wax in filtration and paraffin embedding, samples were washed three times with clearing agent, xylene, at room temperature, with each wash lasting $30 \mathrm{~min}$. Paraffin-embedded tissue samples were then cut into $5-\mu$ m-thick sections and stained at room temperature with Masson's trichrome (Trichrome Stain (Masson) kit, HT15, Sigma-Aldrich; Merck KGaA). This staining technique is used for the detection of collagen fibers (24) in the arterial wall of anterior circulation of brain. The brain tissue slides were immersed in Bouin's solution at $56^{\circ} \mathrm{C}$ for 15 min after deparaffinization and rehydration. The brain sections were stained for $5 \mathrm{~min}$ in Weigert's hematoxylin at room temperature and then washed with tap water. The slides were stained in Biebrich scarlet-acid fuchsin for $5 \mathrm{~min}$, rinsed in distilled water, incubated in phosphotungstic-phosphomolybdic acid for $5 \mathrm{~min}$, dyed with aniline blue for $5 \mathrm{~min}$ and fixed in $1 \%$ acetic acid for $2 \mathrm{~min}$ at room temperature. After rinsing in distilled water, the slides were dehydrated and mounted. The cross-sectional diameter of the arterial lumen was measured from the outermost edge of the lumen from left to right and top to bottom. The average value of these measurements was considered the lumen diameter. All sections were observed and captured under an Olympus BX50 light microscope using an Olympus DP73 camera (Olympus Corporation; magnification, x600). Lumen diameter of the arteries was measured using cellSens software (v1.16, Olympus Corporation).

Western blot analysis of VEGFA and Ang-1. The brain tissue lysates of control, CGM, STZ, STZ + GM and $\mathrm{STZ}+\mathrm{GL}$ rats at 8 weeks were prepared using ice-cold RIPA buffer (Sigma-Aldrich; Merck KGaA) supplemented with $1 \mathrm{X}$ protease inhibitor cocktail (MilliporeSigma). Homogenates were centrifuged at $14,000 \mathrm{x} g$ for $30 \mathrm{~min}$ at $4^{\circ} \mathrm{C}$ to collect the supernatants. The protein concentration of the supernatant was subsequently determined using a BCA protein assay kit (Pierce; Thermo Fisher Scientific, Inc.). Total protein $(10 \mu \mathrm{g})$ was diluted $(1: 2)$ in treatment buffer $(0.125 \mathrm{M}$ Tris-Cl, 4\% SDS, 20\% glycerol, 10\% 2-mercaptoethanol and 
Table I. Comparison of blood glucose levels in different groups.

\begin{tabular}{|c|c|c|c|c|c|}
\hline Week & $\begin{array}{l}\text { Control } \\
(\mathrm{mg} / \mathrm{dl})\end{array}$ & $\begin{array}{c}\text { CGM } \\
(\mathrm{mg} / \mathrm{dl})\end{array}$ & $\begin{array}{c}\text { STZ } \\
(\mathrm{mg} / \mathrm{dl})\end{array}$ & $\begin{array}{c}\mathrm{STZ}+\mathrm{GM} \\
(\mathrm{mg} / \mathrm{dl})\end{array}$ & $\begin{array}{c}\mathrm{STZ}+\mathrm{GL} \\
(\mathrm{mg} / \mathrm{dl})\end{array}$ \\
\hline 1 & $91.8 \pm 3.9$ & $104.3 \pm 4.0$ & $255.7 \pm 4.8^{a}$ & $368.3 \pm 14.2^{\mathrm{a}, \mathrm{c}}$ & $207.3 \pm 19.0^{\mathrm{a}}$ \\
\hline 2 & $99.7 \pm 4.9$ & $106.8 \pm 10.5$ & $348.3 \pm 31.9^{\mathrm{a}}$ & $395.5 \pm 24.8^{\mathrm{a}, \mathrm{b}}$ & $199.3 \pm 35.9^{b}$ \\
\hline 3 & $104.2 \pm 2.6$ & $109.7 \pm 2.8$ & $541.2 \pm 22.6^{a}$ & $318.8 \pm 39.4^{\mathrm{a}, \mathrm{b}}$ & $166.5 \pm 29.6^{b}$ \\
\hline 4 & $96.2 \pm 2.6$ & $106.0 \pm 2.2$ & $383.3 \pm 53.3^{\mathrm{a}}$ & $247.0 \pm 40.3^{\mathrm{a}, \mathrm{b}}$ & $254.0 \pm 19.3^{c}$ \\
\hline 5 & $103.0 \pm 2.1$ & $96.3 \pm 2.5$ & $457.2 \pm 27.0^{\mathrm{a}}$ & $230.3 \pm 44.3^{\mathrm{a}, \mathrm{b}}$ & $190.7 \pm 14.3^{b}$ \\
\hline 6 & $102.5 \pm 2.3$ & $105.2 \pm 1.8$ & $418.5 \pm 13.1^{\mathrm{a}}$ & $203.3 \pm 44.9^{\mathrm{a}, \mathrm{b}}$ & $177.7 \pm 12.2^{b}$ \\
\hline 7 & $96.7 \pm 2.3$ & $110.3 \pm 2.6$ & $439.0 \pm 16.8^{a}$ & $273.2 \pm 44.5^{\mathrm{a}, \mathrm{b}}$ & $121.2 \pm 10.4^{b}$ \\
\hline 8 & $89.2 \pm 3.7$ & $92.7 \pm 2.4$ & $413.2 \pm 11.5^{\mathrm{a}}$ & $211.0 \pm 53.0^{\mathrm{a}, \mathrm{b}}$ & $106.2 \pm 9.7^{b}$ \\
\hline
\end{tabular}

Data are presented as the mean $\pm \mathrm{SEM} ; \mathrm{n}=10$ rats/group. ${ }^{\mathrm{a}} \mathrm{P}<0.001 \mathrm{vs}$. control; ${ }^{b} \mathrm{P}<0.001$ and ${ }^{\mathrm{c}} \mathrm{P}<0.01 \mathrm{vs}$. STZ. CGM, gymnemic control; GL, glibenclamide; GM, gymnemic acid; STZ, streptozotocin. The normal glucose level range of control group is $90-110 \mathrm{mg} / \mathrm{dl}$.

$0.2 \%$ bromophenol blue) and heated for $5 \mathrm{~min}$ at $95^{\circ} \mathrm{C}$. Protein samples were subjected to $12 \%$ SDS-PAGE $(100 \mathrm{~V}, 0.35 \mathrm{~A}$ and $300 \mathrm{~W}$ for $90 \mathrm{~min}$ ) and subsequently transferred onto nitrocellulose membranes (GE Healthcare). Membranes were then blocked with 5\% non-fat dry milk in $0.1 \%$ Tris-buffered saline with Tween ${ }^{\circledR}-20$ (TBS-T) for $1 \mathrm{~h}$ at room temperature. The membranes were incubated with primary antibodies against VEGFA (1:1,000; rabbit polyclonal antibody; cat. no. ab9570; Abcam) Ang-1 (1:1,000; rabbit polyclonal antibody; cat. no. PA1-32150; Thermo Fisher Scientific, Inc.) and $\beta$-actin $(1: 3,000$; rabbit polyclonal antibody; cat. no. ab8227; Abcam) at $4^{\circ} \mathrm{C}$ for $24 \mathrm{~h}$. Membranes were washed three times with TBS-T, followed by incubation for $2 \mathrm{~h}$ at room temperature with goat anti-rabbit horseradish peroxidase-conjugated immunoglobulin G secondary antibodies (1:5,000; ab6721; Abcam). Protein bands were visualized using an enhanced chemiluminescence (ECL) (Pierce ${ }^{\mathrm{TM}}$ ECL Western Blotting Substrate; Thermo Fisher Scientific, Inc.) detection system with Amersham Hyperfilm ${ }^{\mathrm{TM}}$ ECL (GE Healthcare). Resulting bands were subjected to densitometric analysis using Scion Image 4.0 software (Scion Corporation).

Vascular corrosion casting technique. Vascular corrosion casting was performed via an intravascular injection of $0.5 \mathrm{ml}$ heparin $(5,000 \mathrm{IU} / \mathrm{ml}$; Leo Pharma UK/IE), which was immediately injected into the left ventricle to prevent blood clotting. The right atrium functioned as the outlet for blood and injected fluid. A second injection of 400-500 $\mathrm{ml} \mathrm{0.9 \%}$ normal saline solution (General Hospital Products Public Co., Ltd.) was administered to remove the blood from the vascular bed. Subsequently, PU4ii polyurethane-based casting resin (vasQtec) was immediately injected into the cannula through the ascending aorta until reflux from the venous vessels became evident. Each rat was left at room temperature to allow the casting medium to settle for $2 \mathrm{~h}$. The brain was subsequently excised and immersed in water at $80^{\circ} \mathrm{C}$ to complete the hardening process. Thereafter, the brain underwent a corrosive process by adding $10 \% \mathrm{KOH}$ solution (Vidhyasom Co., Ltd.) at room temperature for 1 month. Tissues were removed and washed three times with distilled water. The brain vascular cast was then dissected and were assessed using a stereomicroscope (OLYMPUS SZ2 series) to obtain small specimens and air dried for 2 weeks. The vascular cast of the brain was placed on a metal stub with double side adhesive tape, sprayed with carbon paint and coated with gold on a sputtering apparatus prior to preparation for scanning electron microscopy observation (JEOL JSM-5400; JEOL, Ltd.) at 10-15 KV. The diameter of brain blood vessels was measured using SemAfore 5.2 software (JEOL, Ltd.).

Statistical analysis. Statistical analysis was performed using GraphPad Prism 8.0 (GraphPad Software, Inc.). One-way ANOVA followed by Bonferroni post-hoc analysis was used. Each experiment was repeated at least twice and data are presented as the mean \pm SEM. $\mathrm{P}<0.05$ was considered to indicate a statistically significant difference.

\section{Results}

Effect of GM on blood glucose levels. The effect of GM on blood glucose levels in diabetic rats is presented in Table I. The blood glucose levels in the control, CGM, STZ, STZ + GM and STZ + GL groups were compared at 8 weeks. The results demonstrated that blood glucose levels were significantly elevated in the STZ, STZ + GM and STZ + GL groups compared with the control rats $(\mathrm{P}<0.001)$. Furthermore, blood glucose levels of STZ + GM rats and STZ + GL rats significantly decreased from week 3 to week 8 compared with STZ rats $(\mathrm{P}<0.01$ and $\mathrm{P}<0.001)$.

Effect of GM on body weight. The effect of GM on the body weight of diabetic rats was evaluated in Table II. The results demonstrated that body weight was significantly lower in the STZ group compared with the control group $(\mathrm{P}<0.001)$. After supplementation of GM, body weight was significantly increased every week in the STZ + GM group compared with the STZ group $(\mathrm{P}<0.01$ and $\mathrm{P}<0.001)$.

Masson's trichome histological observation of arteries in the anterior cerebral circulation. Rat brains were histologically analyzed for ICA, MCA and ACA wall thickness, as well as arterial stenosis (Fig. 2). The results demonstrated that in 
Table II. Comparison of body weight in different groups.

\begin{tabular}{|c|c|c|c|c|c|}
\hline Week & Control (g) & CGM (g) & STZ (g) & STZ+GM (g) & STZ+GR (g) \\
\hline 1 & $310.3 \pm 8.4$ & $322.2 \pm 6.7$ & $213.3 \pm 15.5^{\mathrm{a}}$ & $284.4 \pm 14.3^{c}$ & $229.7 \pm 10.1^{\mathrm{a}}$ \\
\hline 2 & $327.2 \pm 9.2$ & $336.7 \pm 5.7$ & $212.2 \pm 19.5^{\mathrm{a}}$ & $294.4 \pm 35.6^{c}$ & $233.3 \pm 10.9^{a}$ \\
\hline 3 & $340.7 \pm 8.4$ & $356.7 \pm 6.3$ & $227.6 \pm 20.2^{\mathrm{a}}$ & $311.3 \pm 16.9^{c}$ & $329.4 \pm 23.3^{c}$ \\
\hline 4 & $351.4 \pm 8.5$ & $369.2 \pm 7.3$ & $230.0 \pm 20.1^{\mathrm{a}}$ & $317.5 \pm 18.7^{c}$ & $267.8 \pm 12.8^{a}$ \\
\hline 5 & $364.1 \pm 8.1$ & $386.3 \pm 7.5$ & $239.6 \pm 20.9^{a}$ & $318.0 \pm 21.1^{\mathrm{d}}$ & $299.4 \pm 14.3^{c}$ \\
\hline 6 & $382.9 \pm 9.2$ & $352.5 \pm 6.9$ & $245.6 \pm 21.9^{b}$ & $346.9 \pm 19.2^{\mathrm{d}}$ & $286.1 \pm 14.6^{\mathrm{b}}$ \\
\hline 7 & $402.1 \pm 9.2$ & $418.3 \pm 3.8$ & $286.1 \pm 28.7^{b}$ & $361.3 \pm 19.49^{c}$ & $299.4 \pm 16.7^{b}$ \\
\hline 8 & $409.3 \pm 9.6$ & $432.2 \pm 7.7$ & $255.4 \pm 24.6^{\mathrm{a}}$ & $367.2 \pm 18.7^{\mathrm{d}}$ & $303.3 \pm 17.2^{\mathrm{b}}$ \\
\hline
\end{tabular}

Data are presented as mean $\pm \mathrm{SEM} ; \mathrm{n}=10$ rats/group. ${ }^{\mathrm{a}} \mathrm{P}<0.001$ and ${ }^{\mathrm{b}} \mathrm{P}<0.01 \mathrm{vs}$. control; ${ }^{\mathrm{c}} \mathrm{P}<0.01$ and ${ }^{\mathrm{d}} \mathrm{P}<0.05$ vs. STZ. CGM, gymnemic control; GL, glibenclamide; GM, gymnemic acid; STZ, streptozotocin.

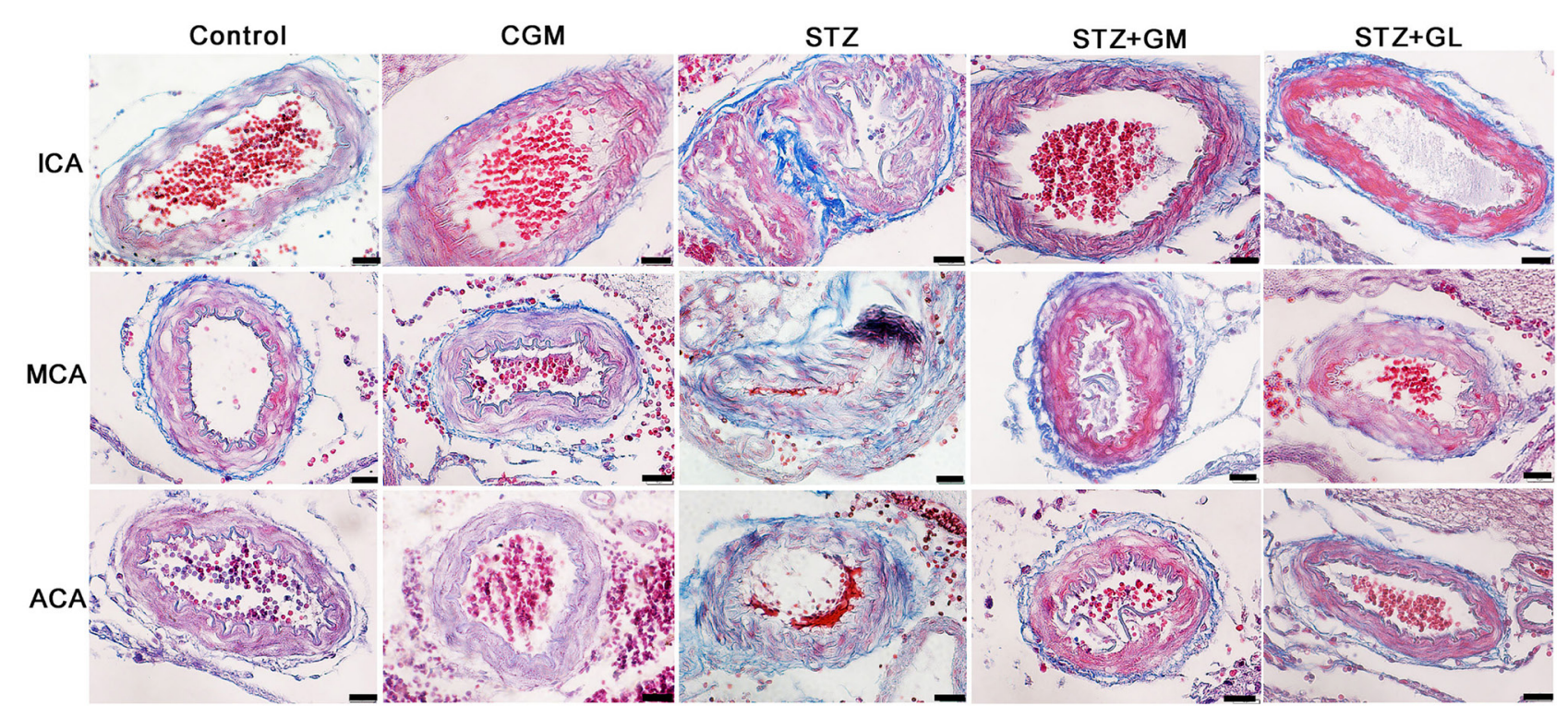

Figure 2. Photomicrographs demonstrating the histological structure of the anterior arteries included in the circle of Willis. Samples obtained from rats in the C, CGM, STZ, STZ + GM and STZ + GL groups were subjected to Masson's trichrome staining (magnification, x600). ACA, anterior cerebral artery; C, control; CGM, gymnemic control; GL, glibenclamide; GM, gymnemic acid; ICA, internal carotid artery; MCA, middle cerebral artery; STZ, streptozotocin.

the STZ group, collagen accumulation (stained in blue) was present in the in tunica media and tunica adventitia of the arterial walls, and a markedly smaller lumen diameter was also demonstrated in the three types of arteries. The arterioles (Fig. 3C) and capillaries (Fig. 4C) exhibited increased collagen thickness in the vessel wall with indications of collagen fibers (blue staining). Decreased collagen fiber accumulation in STZ + GM (Figs. 3D and 4D) and STZ + GL rats (Figs. 3E and 4E was also revealed. The wall thickness of ICA, MCA and ACA arteries were measured and presented in Table III. The wall thickness of each type of artery in the STZ group was significantly increased $(\mathrm{P}<0.001)$ when compared with control and CGM rats. By contrast, they were significantly reduced in $\mathrm{STZ}+\mathrm{GM}$ and $\mathrm{STZ}+\mathrm{GL}$ rats $(\mathrm{P}<0.001$ and $\mathrm{P}<0.01$, respectively) compared with the STZ group. The lumen diameter of the ICA, MCA and ACA was significantly decreased in the STZ group compared with the control and CGM groups (Fig. 5). Conversely, the lumen diameters were increased in STZ + GM and STZ + GL rats compared with the STZ group (Fig. 5).
Vascular corrosion cast examination. Vascular corrosion casts of the anterior circulation of the circle of Willis in control, CGM, STZ, STZ + GM and STZ + GL rats were assessed using a stereomicroscope at low magnification (Fig. 6A). The results demonstrated that the size of the brain vascular cast in the STZ group is smaller and exhibited a greater dropout of vessel density (Fig. 6A) compared with the other groups. The cerebral arterial circle of Willis in the control group exhibited anastomosing branches at the ventral view of the brain in the low magnification SEM scan (Fig. 6B). It formed a polygon surrounding the optic chiasm, optic tracts, pituitary stalk and basal hypothalamus by two ICAs, and the rostral branches (referred to as the posterior cerebral of basilar artery). The anterior and posterior circulation of the polygon was completed by the anastomotic branches (the anterior and posterior communicating arteries) (4). According to the anterior circulation of circle of Willis, the ACAs, which are branches of the two internal carotid arteries, run medially and rostrally downward in front of the optic chiasm. The anterior part of 


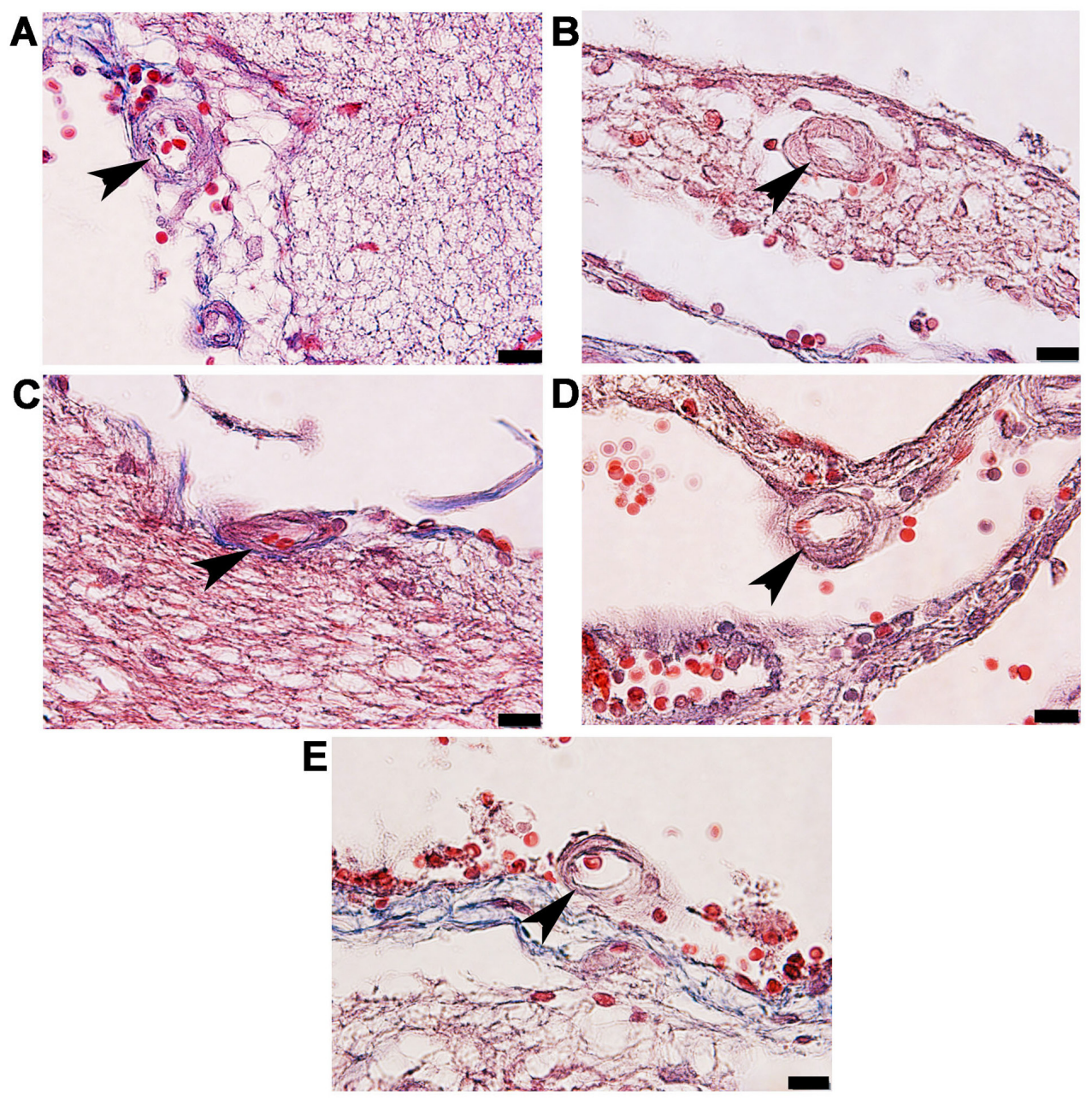

Figure 3. Photomicrographs of the histological structure of arterioles (arrowheads) within the anterior circulation of the circle of Willis. Samples obtained from the (A) control, (B) gymnemic control, (C) STZ, (D) STZ + gymnemic acid and (E) STZ + glibenclamide groups were subjected to Masson's trichrome staining; magnification, x100. STZ, streptozotocin.

the hypothalamus is supplied by the branches of the anterior cerebral and anterior communicating arteries (25). The MCAs are extensive branches of ICAs. They continue into the lateral sulcus where it then branches and projects to several sections of the cerebral hemisphere. The relative diameter of arteries from the anterior circulation of the circle of Willis among the control, CGM, STZ, STZ + GM and STZ + GL groups at 8 weeks were demonstrated ventrally within the brain. Marked shrinkage of the ICA, ACA and MCA was exhibited in the STZ group compared with the other groups (Fig. 7C). At high magnification, the vascular cast of the small blood vessels in the control and CGM groups exhibited the typical pattern of arterioles and capillaries in the hypothalamus and were surrounded by branches of the circle of Willis (Fig. 8A and B). Conversely, the injured blood vessels presented with smaller diameters, stenosis and shrinkage of the arterioles and capillaries in the STZ group (Fig. 8C).

Signs of vessel restoration were demonstrated by increased healthy and nourished ICA, ACA and MCA in the STZ + GM and STZ + GL groups (Fig. 7D and E, respectively). ACA and MCA diameters were significantly decreased in the STZ group compared with the control $(\mathrm{P}<0.001)$ and CGM $(\mathrm{P}<0.01)$ groups; however, no significant differences were observed between the STZ + GM and STZ + GL groups (Fig. 9).
Furthermore, the STZ arterioles from the circle of Willis in the hypothalamus exhibited decreased diameter sizes compared with all other experimental groups (Fig. 10). The same effect was demonstrated in capillaries, although it was not significant. The diameter of arterioles in the STZ group were significantly decreased $(\mathrm{P}<0.001)$ when compared with the control and CGM groups (Fig. 10). Following supplementation with GM and GL, the well-organized architecture of the arteries was restored in the STZ + GM (Fig. 8D) and STZ + GL group (Fig. 8E). Signs of vessel restoration and improvement were exhibited by increased diameters, as well as healthy and nourished arterioles and capillaries in the hypothalamus of the STZ + GM and STZ + GL groups. Additionally, the diameter of arterioles was significantly increased in STZ + GM and $\mathrm{STZ}+\mathrm{GM}$ groups compared with STZ rats $(\mathrm{P}<0.001)$.

Western blot analysis. The western blotting results demonstrated that the protein expression levels of VEGFA and Ang-1 (Fig. 11A) were significantly increased in STZ rats $(\mathrm{P}<0.001)$ compared with the control and CGM rats, whereas VEGFA and Ang-1 expression levels were decreased in STZ + GM and STZ + GL groups compared with STZ rats. Furthermore, VEGFA and Ang-1 protein expression levels were significantly increased in STZ + GM rats compared with 


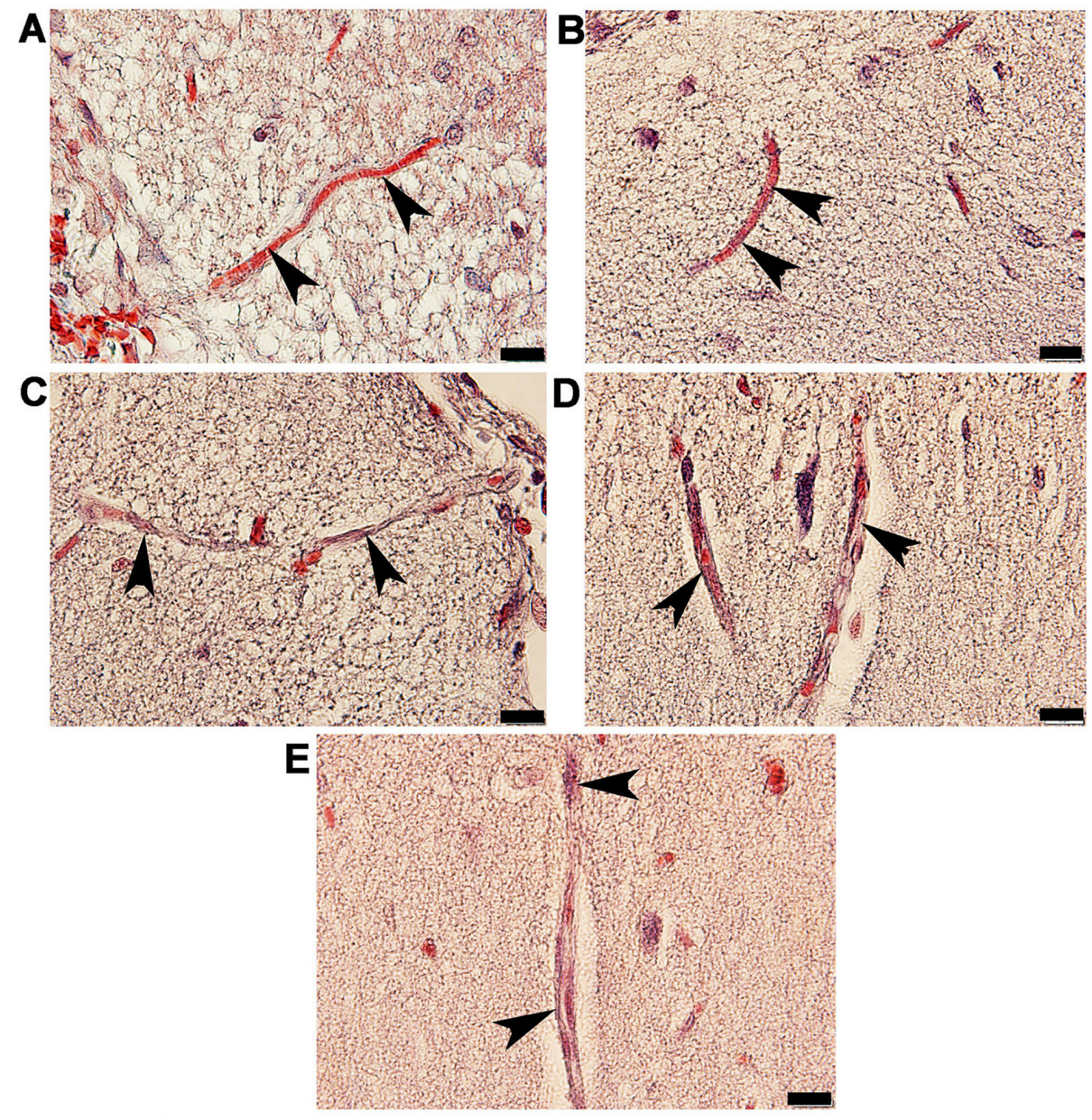

Figure 4. Photomicrographs of the histological structure of capillaries (arrowheads) in the anterior hypothalamus of rats. Samples obtained from the in the (A) control, (B) gymnemic control, (C) STZ, (D) STZ + gymnemic acid and (E) STZ + glibenclamide groups were subjected to Masson's trichrome staining; magnification, x100. STZ, streptozotocin.

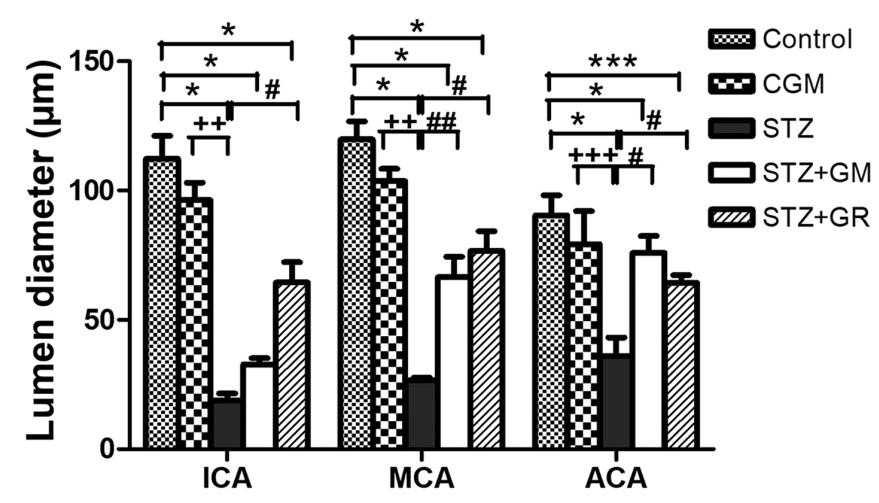

Figure 5. Average lumen diameters of the anterior blood vessels included in the circle of Willis. Tissues collected from the C, CGM, STZ, STZ + GM and STZ + GL groups were subjected to Masson's trichrome staining following 8 weeks of treatment. ${ }^{*} \mathrm{P}<0.001$ and ${ }^{* * *} \mathrm{P}<0.01$ vs. the control group, ${ }^{\#} \mathrm{P}<0.001$ and ${ }^{\# \#} \mathrm{P}<0.01$ vs. STZ; ${ }^{++} \mathrm{P}<0.01$ and ${ }^{++} \mathrm{P}<0.05$ vs. CGM. ACA, anterior cerebral artery; C, control; CGM, gymnemic control; GL, glibenclamide; GM, gymnemic acid; ICA, internal carotid artery; MCA, middle cerebral artery; STZ, streptozotocin.

the control and CGM $(\mathrm{P}<0.0001$ and $\mathrm{P}<0.001)$. However, the protein expression levels of VEGFA and Ang-1 were significantly decreased in STZ + GM rats compared with STZ rats $(\mathrm{P}<0.001$; Fig. 11).

\section{Discussion}

In the present study, the ability of GM to attenuate the diabetes-induced morphological and histopathological alterations of blood vessels in the anterior cerebral circulation of rats was examined. The results demonstrated significantly decreased blood glucose levels in the STZ rats treated with $400 \mathrm{mg} / \mathrm{kg} \mathrm{GM}$ for a total period of 8 weeks compared with untreated diabetic groups. This result is consistent with previous studies, which have indicated that GS extracts may possess antidiabetic properties $(16,23)$. The antidiabetic and antioxidative properties may be due to a number of bioactive compounds present in GS extracts, including oleanane-type triterpenoid saponins (known as GM), alkaloids, acidic glycosides and anthraquinones and their derivatives (26).

In the present study, histological analysis by Masson's trichrome staining of ICA, MCA and ACA from the brain tissues of STZ rats demonstrated wall thickening and increased collagen fiber deposition in the tunica media and tunica adventitia of arteries. Furthermore, increased collagen thickness was demonstrated in the walls of arterioles and capillaries. Moreover, STZ rats exhibited lumen narrowing in each type of artery. Collagen deposition can lead to the stenosis of arteries, whereas immoderate collagen breakdown combined with insufficient synthesis weakens plaques, thereby making 
A

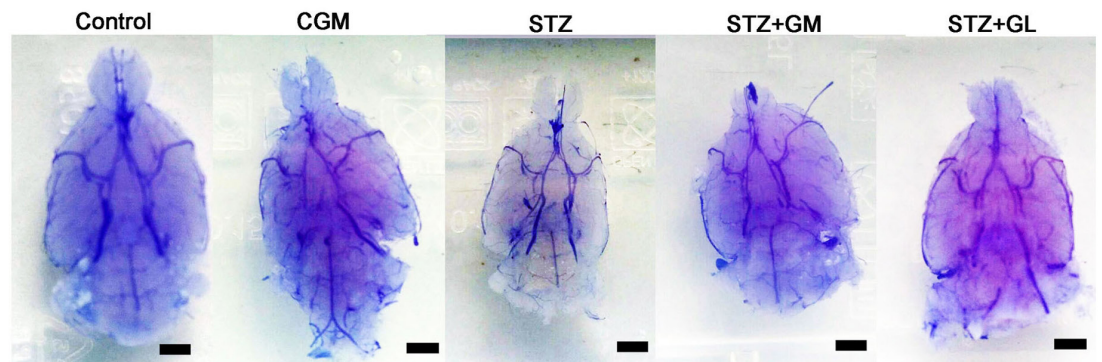

B

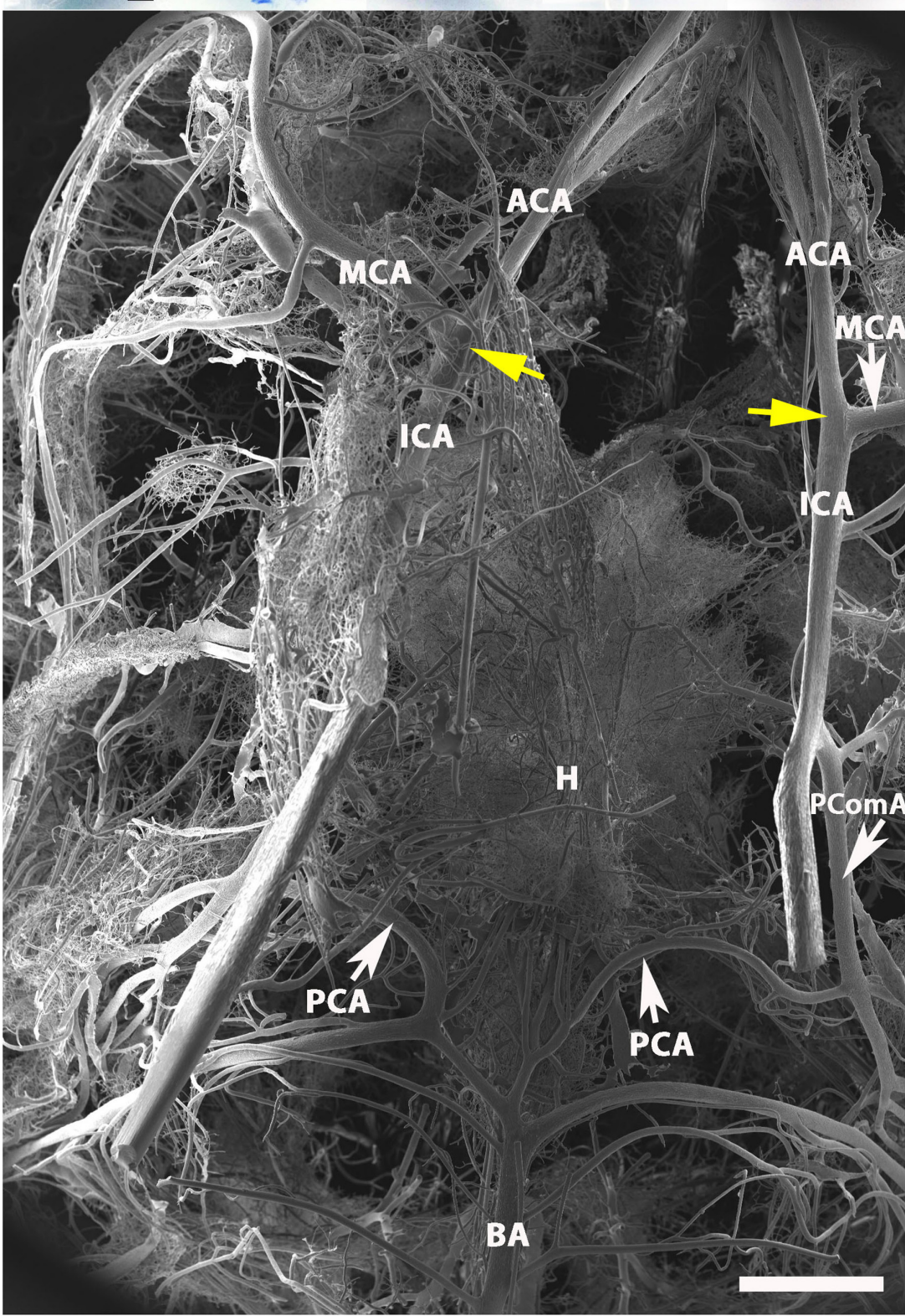

Figure 6. Vascular corrosion casts of the brain of Wistar rats. (A) Stereomicrographs of the ventral views of the brain vascular corrosion cast in control, CGM, STZ, STZ + GM and STZ + GL rats showing the branches from circle of Willis (scale bar, $1 \mathrm{~mm}$ ). (B) Low magnification scanning electron micrograph of the circle of Willis in a representative control group rat. The image presents the ventral view of the brain. The circle of Willis is a circulatory anastomosis (yellow arrows) that supplies blood to the brain. A ring is formed surrounding the basal hypothalamus. ACA, anterior cerebral artery; BA, basilar artery; CGM, gymnemic control; GL, glibenclamide; GM, gymnemic acid; H, hypothalamus; ICA, internal carotid artery; MCA, middle cerebral artery; PCA, posterior cerebral artery; PComA, posterior communicating artery; STZ, streptozotocin (scale bar, $1 \mathrm{~mm}$ ).

them rupture (27). Newly synthesized collagen may work as a substrate leading to luminal narrowing (27). Arterial stenosis is the narrowing of large arteries that transfer blood to the body, including the head, face and brain (28). The destruction of the vascular wall results in vascular complications in diabetes (28). Early structural impairment of the common carotid artery is often associated with type I diabetes and is considered an early sign of cerebral atherosclerosis, which is also reflected by the increased wall thickness of the tunica intima and tunica media (29). These histopathological changes result in blood vessels becoming inflexible, thus becoming unable to react to exogenous or endogenous stimuli to effectively regulate blood 

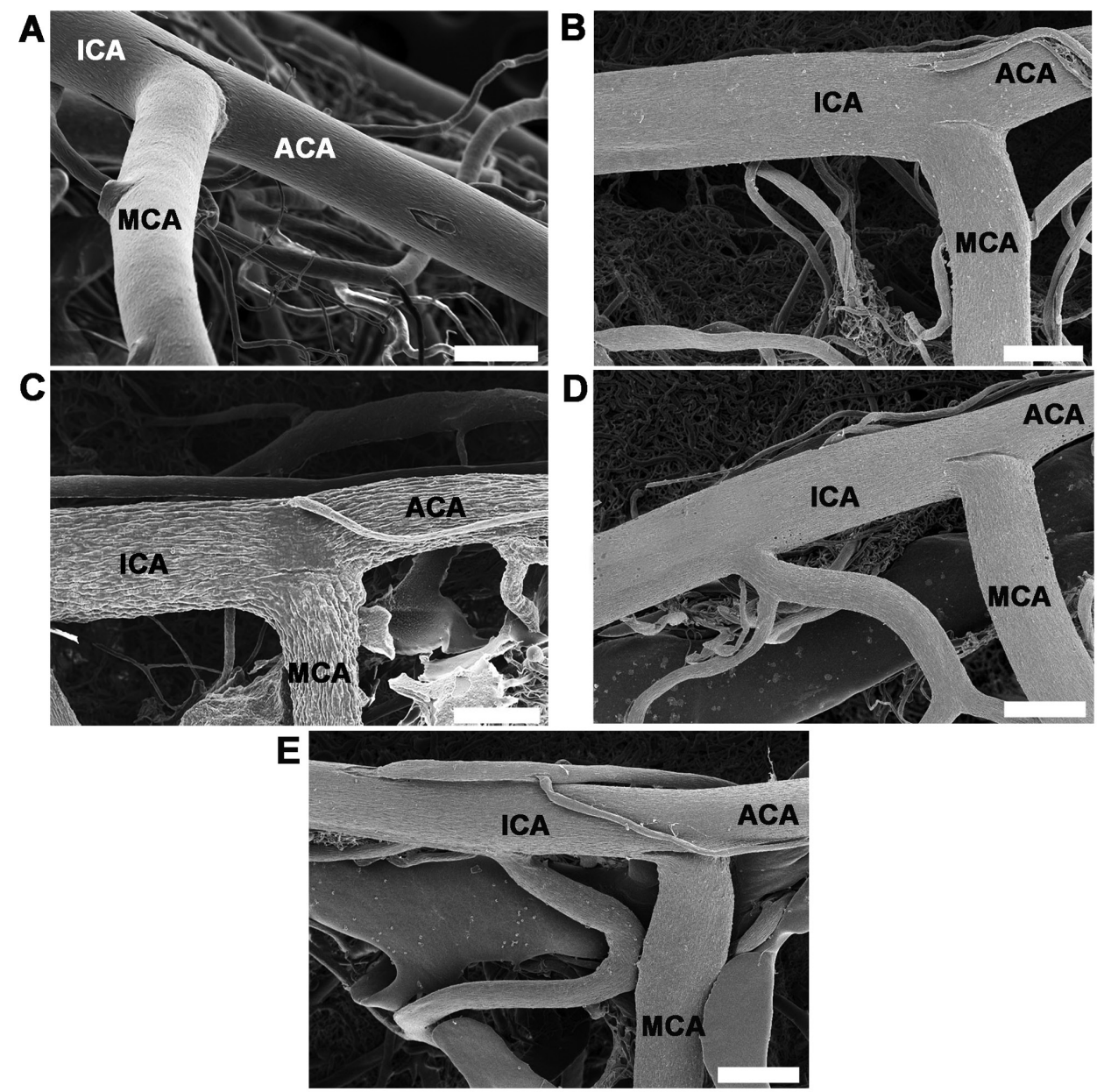

Figure 7. High magnifications of vascular corrosion casts of the anterior circulatory anastomosis of the brain. Scanning electron micrographs of the ventral views of the ICA, ACA and MCA from anterior cerebral circulation of circle of Willis in (A) control, (B) gymnemic control, (C) STZ, (D) STZ + GM and (E) STZ + GL rats; scale bars, $200 \mu \mathrm{m}$. The STZ group demonstrated a marked shrinkage of the ICA, ACA and MCA. Signs of vessel restoration and improvement were exhibited by the increasingly healthy and nourished blood vessels of the STZ + GM and STZ + GL groups. ACA, anterior cerebral artery; GL, glibenclamide; GM, gymnemic acid; ICA, internal carotid artery; MCA, middle cerebral artery; STZ, streptozotocin.

flow (2). Atherosclerosis is a regressive blood vessel disorder that generally affects medium to large-sized arteries. In the brain, the vessels of the circle of Willis are often affected byy atherosclerosis (30).

Vascular corrosion casting demonstrated significant destruction of arteries (ICA, MCA and ACA) and small vessels (arterioles and capillaries) in the brains of the STZ group. Vessel diameter is a key determinant of stroke extension; under normal physiological conditions, vessel diameter is regulated by several pathways, such as intracellular signaling, cytokine and nervous control that ensure adequate blood flow (30). Following cerebral artery occlusion, the consecutive drop in local CBF is offset by recruitment of the collateral artery and vasodilation, thus limiting the extension of the lesion; this effect is mediated by nitric oxide (NO) (31). VEGF mediates the endothelial nitric oxide synthase (eNOS) expression of NO following cerebral ischemia (31). Physical activity improves long-term stroke outcome through eNOS-dependent mechanisms that increase angiogenesis and CBF (32). In the vascular system, VEGF and Ang-1 (with its receptor Tie2), are essential angiogenic factors that control angiogenesis between small and large blood vessels (33). VEGF serves a crucial role in angiogenesis and neuronal regeneration following ischemic stroke (34). VEGF is a master regulator of angiogenesis that has been shown to directly regulate lumen size. Changes in lumen diameter occur in response to blood pressure and blood flow, and subsequently control oxygen delivery and immunological surveillance. VEGF and Ang-1 are highly effective at inducing endothelial proliferation and promoting hyperplasia, tortuosity and decreased lumen diameter. Therefore, increased expression of these two proteins results in the narrowing of the lumen diameter (35).

In chronic and weak cerebral hypoxia demonstrated in patients with large cerebral artery stenosis, VEGF expression may increase prior to the onset of a stroke and may function as an angiogenic molecule (36). However, VEGF has been reported to also cause leakage of the blood-brain barrier, inflammation and brain edema (37). Ang-1 modulates the maturation and stabilization of newly formed vessels, including adherence and maintaining the survival of vascular epithelial cells (38). Ang-1 decreases permeability of the endothelial cell and increases vascular stabilization by recruiting pericytes and smooth muscle cells to the growing blood vessels. Together, VEGF and Ang-1 enhance angiogenesis and are more effective in decreasing blood-brain barrier leakage compared with VEGF alone (39).

In the present study, western blotting demonstrated that VEGF and Ang-1 protein expression levels were increased in 

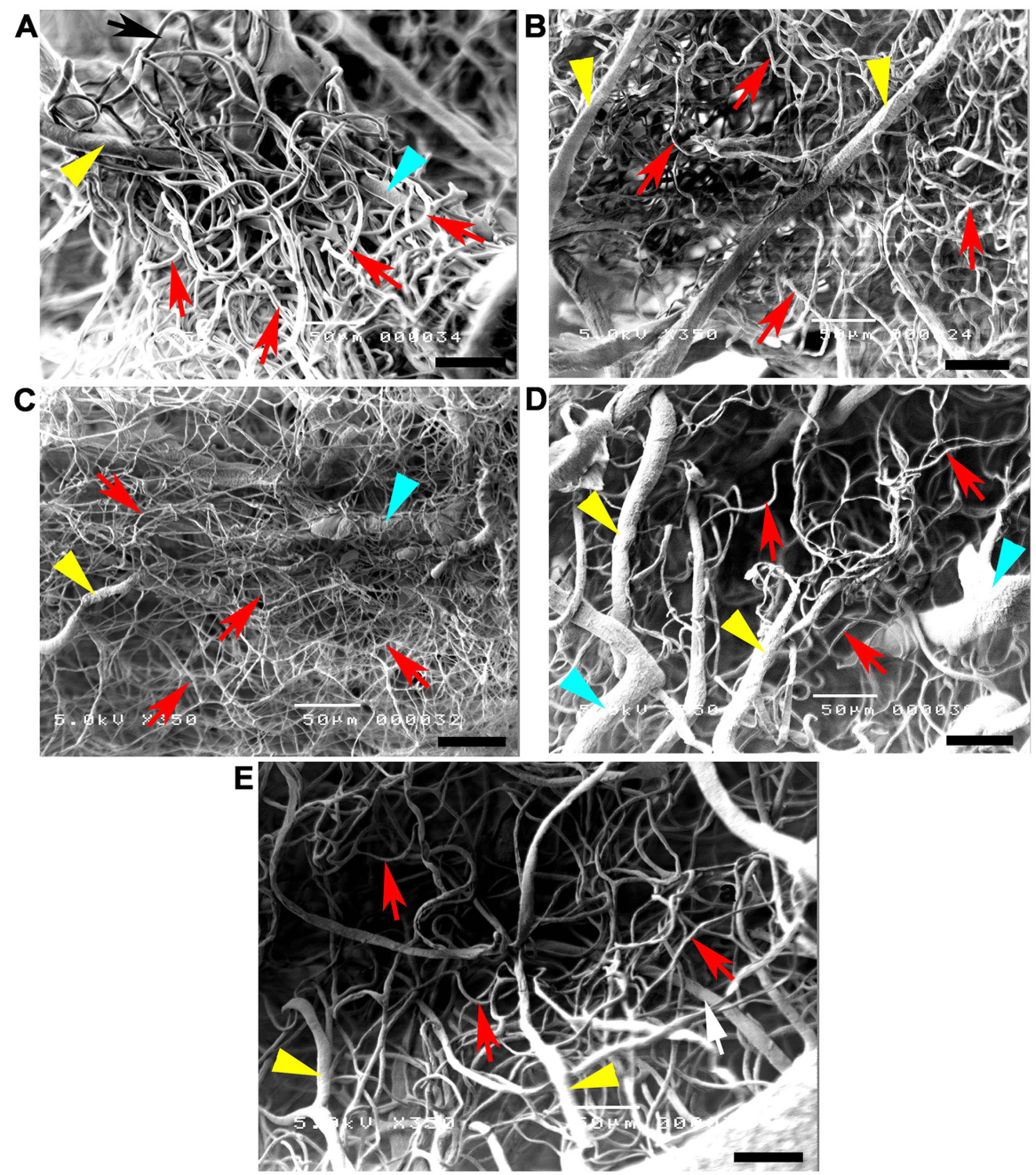

Figure 8. High magnification scanning electron micrographs of hypothalamic vascular casts at 8 weeks. (A) Control and (B) gymnemic control rats exhibited healthy small blood vessels with branching arterioles and venules, which joined further into numerous capillaries, forming a dense capillary meshwork. (C) Rats in the STZ group presented with critical arteriole and capillary shrinkage. Rats in the (D) STZ + gymnemic acid and (E) the STZ + glibenclamide groups exhibited arteriole and capillary recovery and redevelopment. Scale bars, $50 \mu \mathrm{m}$. yellow arrowheads, arterioles; STZ, streptozotocin; green arrowheads, venules; red arrows, capillaries.

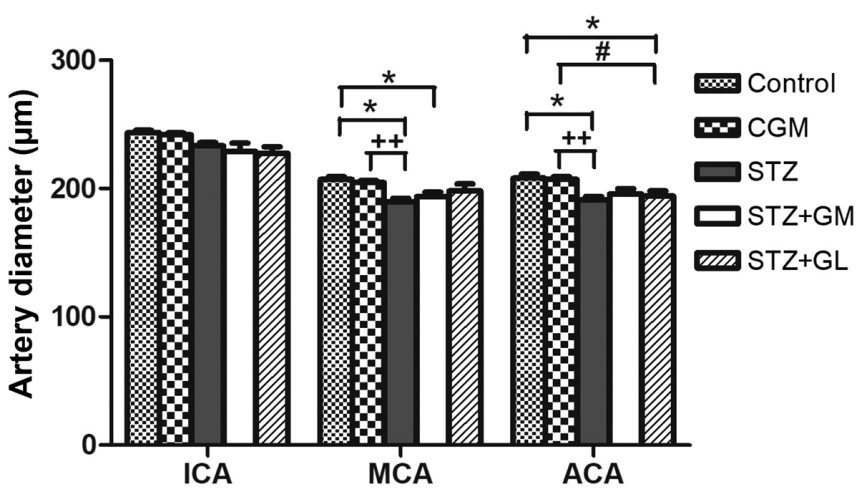

Figure 9. Average diameter of arteries from the anterior circulation of the circle of Willis in control, CGM, STZ, STZ + GM and STZ + GL rats at 8 weeks. Data are presented as the mean \pm SEM. ${ }^{*} \mathrm{P}<0.001$ vs. control; ${ }^{\#} \mathrm{P}<0.001$ vs. STZ; ${ }^{++} \mathrm{P}<0.01$ vs. CGM. ACA, anterior cerebral artery; CGM, gymnemic control; GL, glibenclamide; GM, gymnemic acid; ICA, internal carotid artery; MCA, middle cerebral artery; STZ, streptozotocin.

the STZ group compared with the control group. This angiogenic factors are increased in cerebral microvessels of diabetic

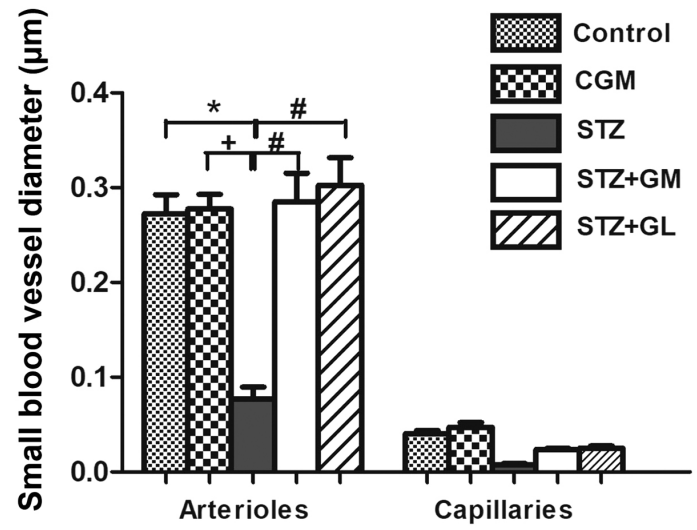

Figure 10. Average diameter of small blood vessels from branches of the circle of Willis in the hypothalamus of control, CGM, STZ, STZ + GM and STZ + GL rats at 8 weeks. Data are presented as the mean \pm SEM ${ }^{*} \mathrm{P}<0.001$ vs. control; ${ }^{\# P}<0.001$ vs. $\mathrm{STZ} ;{ }^{+} \mathrm{P}<0.01$ vs. CGM. CGM, gymnemic control; GL, glibenclamide; GM, gymnemic acid; STZ, streptozotocin.

animals is consistent with previous results on other diabetic animal models, such as high-fat-diet, Goto-Kakizaki rats and 

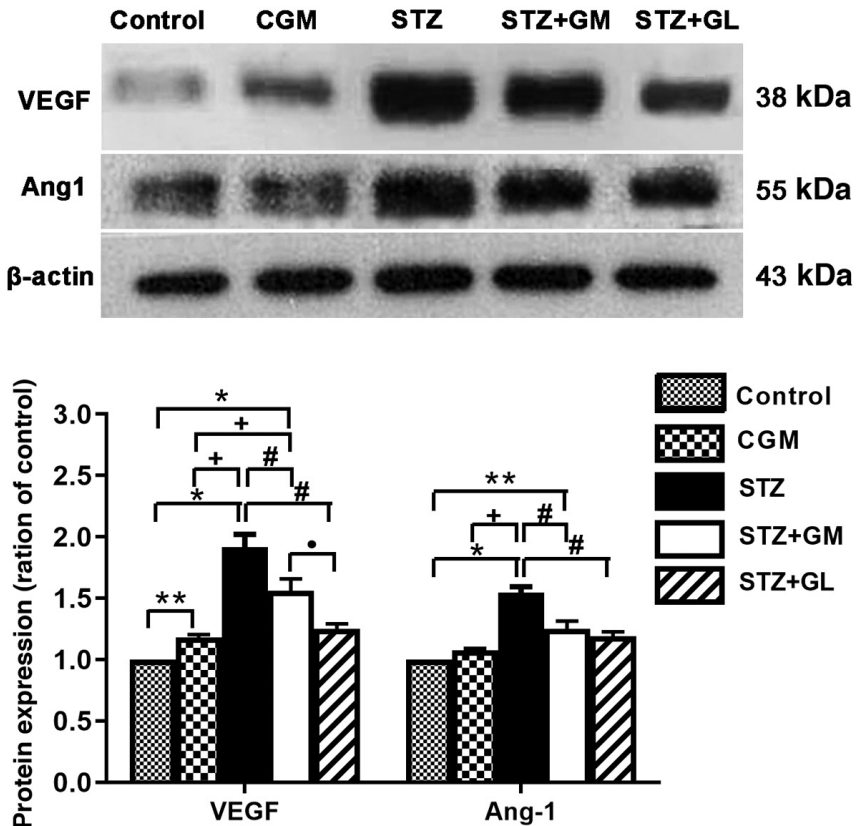

Figure 11. Densitometric analysis of VEGF and Ang-1 protein expression in the hypothalamus of control, CGM, STZ, STZ + GM, and $\mathrm{STZ}+\mathrm{GL}$ rats at 8 weeks. Data are presented as the mean \pm SEM ${ }^{*} \mathrm{P}<0.001$ and ${ }^{* *} \mathrm{P}<0.01$ vs. control; ${ }^{*} \mathrm{P}<0.001$ vs. $\mathrm{STZ}$; ${ }^{+} \mathrm{P}<0.001$ vs. $\mathrm{CGM}$; $\cdot \mathrm{P}<0.001$ vs. STZ+GL. Ang-1, angiopoietin-1; CGM, gymnemic control; GL, glibenclamide; GM, gymnemic acid; STZ, streptozotocin.

$\mathrm{db} / \mathrm{db}$ mice (40). These changes were associated with increased VEGF signaling (41). An increased understanding of the regulation of cerebral neovascularization in diabetes is required to identify preventive and therapeutic strategies against cerebral complications (42). Hypoperfusion of the brain results in hypoxia-induced angiogenesis through the upregulated expression of hypoxia-inducible genes, such as VEGF and Ang-1 (43). Arterial stenosis or atherosclerotic changes of the vessels caused by hypoxia are considered to serve an important role in the pathogenesis of brain ischemia (44). During inflammation, there is a pathological increase in vascular leakage mediated by VEGF (45); Ang-1 has been reported to counteract the VEGF-induced inflammation and vascular leakage in endothelial cells as well as exhibiting an additive effect on vessel formation (4).

GM is the active compound of GS and is found in all parts of the plant (46). GS possesses glucose lowering effects, which are associated with reduced ROS in rats and improved antioxidant parameters, such as like glutathione, glutathione peroxidase, catalase and malondialdehyde (47). GAPDH is a key enzyme in the glycolysis pathway (18); it has been reported to interact with GAPDH and has been indicated to serve an important role in hypoglycemic activity (19). Previous studies have demonstrated that GM inhibits $\mathrm{Na}^{+}$dependent active glucose transport in the small intestine (47) and inhibits glucose-stimulated gastric inhibitory peptide (48). GM molecules prevent glucose absorption in the intestine of rats by filling the receptor location in the absorptive external layers of the intestinal wall (19). Furthermore, GM can inhibit glucose uptake in the intestine of guinea pigs, which occurs from to the suppressive effect of GM on high $\mathrm{K}^{+}$-induced contraction in guinea pig ileal longitudinal muscles (49). Thus, the use of GM can be beneficial for diabetic animals owing to its anti-inflammatory properties along with anti-oxidant potential.

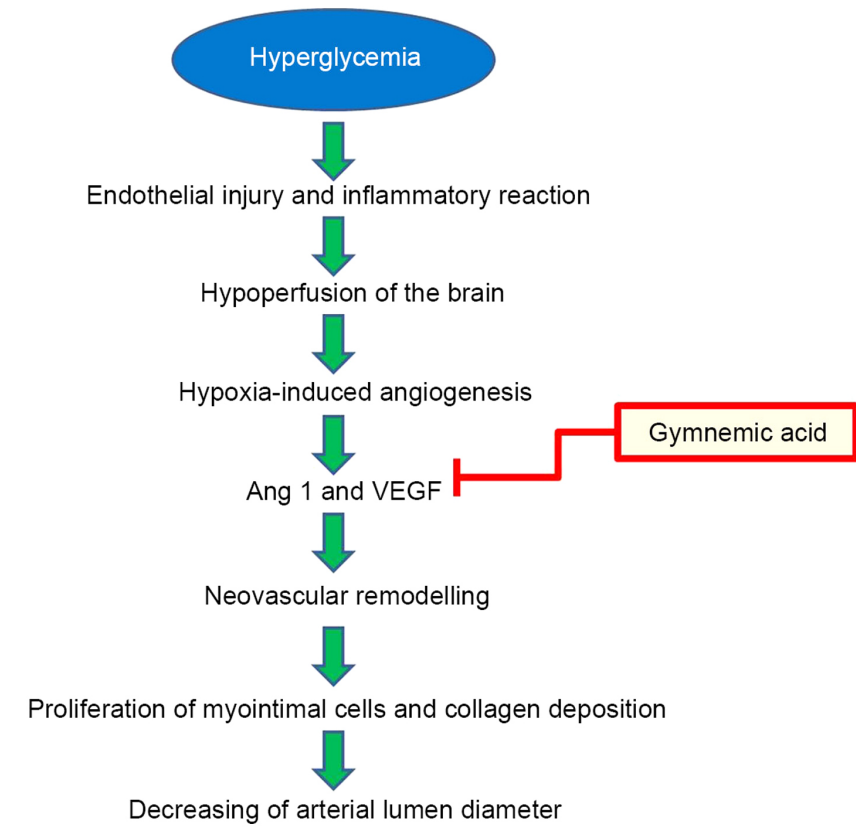

Figure 12. A proposed potential inhibitory mechanism for improvement of the endothelial injury and inflammatory reaction of brain blood vessels in STZ induced-diabetic rats by gymnemic acid. The inner wall of blood vessels impacts high blood glucose conditions, resulting in brain hypoxia-induced angiogenesis and neovascular remodeling by upregulating Ang-1 and VEGF expression. VEGF protein and receptors increase lumen formation and decrease lumen diameter by proliferation of myointimal cells and collagen deposition, and Ang-1 serves important roles in angiogenesis and vascular development. STZ, streptozotocin; VEGF, vascular endothelial growth factor.

Hyperglycemia, a high blood glucose can affect endothelial cells dysfunction of brain blood vessels in STZ induced-diabetic rats, resulting in brain hypoxia-induced angiogenesis by upregulating expression of the hypoxia-inducible gene (50). The beneficial effects of GM treatment $(400 \mathrm{mg} / \mathrm{kg})$ improved the pathological changes in brain blood vessels by reducing neovascular remodeling via the inhibition of VEGF and Ang-1 (Fig. 12). VEGF protein and receptors increase lumen formation and decrease lumen diameter by proliferation of myointimal cells and collagen deposition (51), and Ang-1 serves important roles in angiogenesis and vascular development. Ang-1 has powerful vascular protective effects, such as suppressing plasma leakage, inhibiting vascular inflammation and preventing endothelial death (52).

In the present study, vascular corrosion casting and microscopy demonstrated that treatment with GM in the diabetic rat group recovered the healthy architecture of vessels. GM administration resulted in the improvement and restoration of injured brain blood vessels induced by hyperglycemia. The lumen diameter, wall thickness of arteries and the diameter of arteries, arterioles and capillaries were all improved.

The present study is not without limitations. For example, the study only assessed the effects of GM substances for 8 weeks to determine whether they could restore the small blood vessels of the brain. A longer period of time is required to determine the effective regeneration of large blood vessels.

Taken together, the results of the present study demonstrated that the restoration and improvement of brain vascular characteristics in the hypothalamus, through decreased VEGF expression and Ang-1 secretion, may be beneficial for 
investigating the role of GM as a therapeutic target for stroke following treatment for diabetes.

\section{Acknowledgements}

The authors would like to thank Mrs. Anna Chatthong from International Relations Officer, Faculty of Science, Prince of Songkla University for their assistance with revising the manuscript.

\section{Funding}

The present study was supported by the Prince of Songkla University Research Fund (grant no. SCI610515S).

\section{Availability of data and materials}

The datasets used and/or analyzed during the current study are available from the corresponding author on reasonable request.

\section{Authors' contributions}

WK designed and conducted the research. NS and PB prepared animal tissue and performed staining. WK, NS and RJ performed vascular corrosion casting. NS and MK performed the western blot analysis. WK wrote the manuscript and performed statistical analysis. The authenticity of all raw data was assessed by WK and NS. All authors have read and approved the final version of the manuscript.

\section{Ethics approval and consent to participate}

The experimental protocol used was approved by the Animal Ethics Committee of the Prince of Songkla University (Hat Yai, Songkhla, Thailand).

\section{Patient consent for publication}

Not applicable.

\section{Competing interests}

The authors declare that they have no competing interests.

\section{References}

1. American Diabetes Association: Diagnosis and classification of diabetes mellitus. Diabetes Care 32 (Suppl 1): S62-S67, 2009.

2. Enache AL, Slujitoru AS, Pintea IL, Stocheci CM, Mateescu GO and Gheorghişor I: Histological and immunohistochemical aspects of cerebral vessels of the elderly. Rom J Morphol Embryol 53: 1043-1050, 2012.

3. Naderi A,Zahed R,Aghajanpour L,AmoliFA and Lashay A: Long term features of diabetic retinopathy in streptozotocin-induced diabetic Wistar rats. Exp Eye Res 184: 213-220, 2019.

4. Javed K and Das MJ: Neuroanatomy, Posterior Cerebral Arteries. StatPearls Publishing, Treasure Island, FL, 2020.

5. Mukherjee D, Jani ND, Narvid J and Shadden SC: The role of circle of Willis anatomy variations in cardio-embolic stroke: A patient-specific simulation based study. Ann Biomed Eng 46: 1128-1145, 2018.

6. Yu R and Lui F: Neuroanatomy, Brain Arteries. StatPearls Publishing, Treasure Island, FL, 2019.

7. Shi Y and Vanhoutte PM: Macro- and microvascular endothelial dysfunction in diabetes. J Diabetes 9: 434-449, 2017.
8. Conway EM, Collen D and Carmeliet P: Molecular mechanisms of blood vessel growth. Cardiovasc Res 49: 507-521, 2001.

9. Rodella LF and Favero G: Atherosclerosis and current anti-oxidant strategies for atheroprotection. Available from: https://app.dimensions.ai/details/publication/pub.1024158465.

10. Shibuya M: Vascular endothelial growth factor (VEGF) and its receptor (VEGFR) signaling in angiogenesis: A crucial target for anti- and pro-angiogenic therapies. Genes Cancer 2: 1097-1105, 2011.

11. Aguilera KY and Brekken RA: Recruitment and retention: Factors that affect pericyte migration. Cell Mol Life Sci 71: 299-309, 2014.

12. Wirostko B, Wong TY and Simó R: Vascular endothelial growth factor and diabetic complications. Prog Retin Eye Res 27: 608-621, 2008

13. Akwii RG, Sajib MS, Zahra FT and Mikelis CM: Role of Angiopoietin-2 in vascular physiology and pathophysiology. Cells 8: 471, 2019.

14. Melgar-Lesmes P and Edelman ER: Monocyte-endothelial cell interactions in the regulation of vascular sprouting and liver regeneration in mouse. J Hepatol 63: 917-925, 2015.

15. Thakur G, Sharma R, Sanodiya BS, Pandey M and Bisen P: Gymnema sylvestre: An alternative therapeutic agent for management of diabetes. Pharm Sci Technol Today 2: 1-6, 2012.

16. Ahamad J, Amin S and Mir SR: Simultaneous quantification of gymnemic acid as gymnemagenin and charantin as $\beta$-sitosterol using validated HPTLC densitometric method. J Chromatogr Sci 53: 1203-1209, 2015.

17. El Shafey AAM, El-Ezabi MM, Seliem MME, Ouda HHM and Ibrahim DS: Effect of Gymnema sylvestre R. Br. leaves extract on certain physiological parameters of diabetic rats. J King Saud Univ Sci 25: 135-141, 2013.

18. Tiwari P, Mishra BN and Sangwan NS: Phytochemical and pharmacological properties of Gymnema sylvestre: An important medicinal plant. BioMed Res Int 2014: 830285, 2014.

19. Sahu NP, Mahato SB, Sarkar SK and Poddar G: Triterpenoid saponins from Gymnema sylvestre. Phytochemistry 41: 1181-1185, 1996.

20. Li Y, Sun M, Liu Y, Liang J, Wang T and Zhang Z: Gymnemic acid alleviates type 2 diabetes mellitus and suppresses endoplasmic reticulum stress in vivo and in vitro. J Agric Food Chem 67: 3662-3669, 2019.

21. Nazaruk $\mathbf{J}$ and Borzym-Kluczyk M: The role of triterpenes in the management of diabetes mellitus and its complications. Phytochem Rev 14: 675-690, 2015.

22. Prabhu S and Vijayakumar S: Antidiabetic, hypolipidemic and histopathological analysis of Gymnema sylvestre (R. Br) leaves extract on streptozotocin induced diabetic rats. Biomed Prev Nutr Internet 4: 425-430, 2014.

23. Wu F, Jin Z and Jin J: Hypoglycemic effects of glabridin, a polyphenolic flavonoid from licorice, in an animal model of diabetes mellitus. Mol Med Rep 7: 1278-1282, 2013.

24. Komolkriengkrai M, Nopparat J, Vongvatcharanon U, Anupunpisit V and Khimmaktong W: Effect of glabridin on collagen deposition in liver and amelioration of hepatocyte destruction in diabetes rats. Exp Ther Med 18: 1164-1174, 2019.

25. Cui Y, Xu T, Chen J, Tian $\mathrm{H}$ and Cao H: Anatomic variations in the anterior circulation of the circle of Willis in cadaveric human brains. Int J Clin Exp Med 8: 15005-15010, 2015.

26. Fatani AJ, Al-Rejaie SS, Abuohashish HM, Al-Assaf A, Parmar MY, Ola MS and Ahmed MM: Neuroprotective effects of Gymnema sylvestre on streptozotocin-induced diabetic neuropathy in rats. Exp Ther Med 9: 1670-1678, 2015.

27. Rekhter MD: Collagen synthesis in atherosclerosis: Too much and not enough. Cardiovasc Res 41: 376-384, 1999.

28. Grinberg LT and Thal DR: Vascular pathology in the aged human brain. Acta Neuropathol 119: 277-290, 2010.

29. Chen R, Ovbiagele B and Feng W: Diabetes and stroke: Epidemiology, pathophysiology, pharmaceuticals and outcomes. Am J Med Sci 351: 380-386, 2016.

30. Castilla-Guerra L and Fernandez-Moreno MC: Stroke in diabetic patients: Is it really a macrovascular complication? Stroke 38: e106-e106, 2007.

31. Poittevin M, Bonnin P, Pimpie C, Rivière L, Sebrié C, Dohan A, Pocard M, Charriaut-Marlangue C and Kubis N: Diabetic microangiopathy: Impact of impaired cerebral vasoreactivity and delayed angiogenesis after permanent middle cerebral artery occlusion on stroke damage and cerebral repair in mice. Diabetes 64: 999-1010, 2015. 
32. Yin K-J, Hamblin M and Chen YE: Angiogenesis-regulating microRNAs and ischemic stroke. Curr Vasc Pharmacol 13: 352-365, 2015.

33. Marti HJ, Bernaudin M, Bellail A, Schoch H, Euler M, Petit E and Risau W: Hypoxia-induced vascular endothelial growth factor expression precedes neovascularization after cerebral ischemia. Am J Pathol 156: 965-976, 2000.

34. Zhang Z and Chopp M: Vascular endothelial growth factor and angiopoietins in focal cerebral ischemia. Trends Cardiovase Med 12: 62-66, 2002.

35. Niu G and Chen X: Vascular endothelial growth factor as an anti-angiogenic target for cancer therapy. Curr Drug Targets 11: 1000-1017, 2010

36. Matsuo R, Ago T, Kamouchi M, Kuroda J, Kuwashiro T, Hata J Sugimori H, Fukuda K, Gotoh S, Makihara N, et al: Clinical significance of plasma VEGF value in ischemic stroke - research for biomarkers in ischemic stroke (REBIOS) study. BMC Neurol 13 32, 2013.

37. FagianiE,Lorentz P, Kopfstein L and Christofori G: Angiopoietin-1 and -2 exert antagonistic functions in tumor angiogenesis, yet both induce lymphangiogenesis. Cancer Res 71: 5717-5727, 2011.

38. Akwii RG, Sajib MS, Zahra FT and Mikelis CM: Mikelis Role of Angiopoietin-2 in vascular physiology and pathophysiology. Cells 8: 471, 2019.

39. Peplow PV: Growth factor- and cytokine-stimulated endothelial progenitor cells in post-ischemic cerebral neovascularization. Neural Regen Res 9: 1425-1429, 2014.

40. Prakash R, Somanath PR, El-Remessy AB, Kelly-Cobbs A Stern JE, Dore-Duffy P, Johnson M, Fagan SC and Ergul A: Enhanced cerebral but not peripheral angiogenesis in the Goto-Kakizaki model of type 2 diabetes involves VEGF and peroxynitrite signaling. Diabetes 61: 1533-1542, 2012.

41. Abdelsaid M, Coucha M, Hafez S, Yasir A, Johnson MH and Ergul A: Enhanced VEGF signalling mediates cerebral neovascularisation via downregulation of guidance protein ROBO4 in a rat model of diabetes. Diabetologia 60: 740-750, 2017.

42. Pugh CW and Ratcliffe PJ: Regulation of angiogenesis by hypoxia: Role of the HIF system. Nat Med 9: 677-684, 2003.
43. Pogue AI and Lukiw WJ: Angiogenic signaling in Alzheimer's disease. Neuroreport 15: 1507-1510, 2004.

44. Gamble JR, Drew J, Trezise L, Underwood A, Parsons M, Kasminkas L, Rudge J, Yancopoulos G and Vadas MA: Angiopoietin-1 is an antipermeability and anti-inflammatory agent in vitro and targets cell junctions. Circ Res 87: 603-607, 2000.

45. Thurston G, Suri C, Smith K, McClain J, Sato TN, Yancopoulos GD and McDonald DM: Leakage-resistant blood vessels in mice transgenically overexpressing angiopoietin-1. Science 286: 2511-2514, 1999.

46. Ibrahim A, Onyike E, Nok AJ and Umar IA: Combined effect on antioxidant properties of Gymnema sylvestre and combretum micranthum leaf extracts and the relationship to hypoglycemia. Eur Sci J: Dec 31, 2017 (Epub ahead of print). doi: org/10.19044/ esj.2017.v13n36p266.

47. Yoshioka S: Inhibitory effects of gymnemic acid and an extract from the leaves of Zizyphus jujuba on glucose absorption in the rat small intestine. J Yonago Med Assoc 37: 142-154, 1986.

48. Fushiki T, Kojima A, Imoto T, Inoue K and Sugimoto E: An extract of Gymnema sylvestre leaves and purified gymnemic acid inhibits glucose-stimulated gastric inhibitory peptide secretion in rats. J Nutr 122: 2367-2373, 1992.

49. Shimizu K, Iino A, Nakajima J, Tanaka K, Nakajyo S, Urakawa N, Atsuchi M, Wada T and Yamashita C: Suppression of glucose absorption by some fractions extracted from Gymnema sylvestre leaves. J Vet Med Sci 59: 245-251, 1997.

50. Krock BL, Skuli N and Simon MC: Hypoxia-induced angiogenesis: Good and evil. Genes Cancer 2: 1117-1133, 2011.

51. Xu J and Shi GP: Vascular wall extracellular matrix proteins and vascular diseases. Biochim Biophys Acta 1842: 2106-2119, 2014.

52. Brindle NPJ, Saharinen P and Alitalo K: Signaling and functions of angiopoietin-1 in vascular protection. Circ Res 98: 1014-1023, 2006.

This work is licensed under a Creative Commons Attribution-NonCommercial-NoDerivatives 4.0 International (CC BY-NC-ND 4.0) License. 